\title{
Zur zielgruppengerechten Gestaltung der UWSF
}

\author{
Almut B. Heinrich $\cdot$ Henner Hollert
}

Online veröffentlicht: 29. September 2009

(C) Springer-Verlag 2009

Liebe Leserinnen,

liebe Leser,

durch das höhere Manuskriptaufkommen haben wir in diesem Jahr die Anzahl der Ausgaben von vier auf sechs pro Jahrgang erhöht und konnten UWSF für die Evaluierung durch Thomson ISI Web of Science Extended anmelden. Im Erfolgsfall sind UWSF-Beiträge in Zukunft auch in ISI Web of Science Extended zu finden, und nach einer zweijährigen Evaluierung würde der Impact Factor von UWSF berechnet.

Um auch vor diesem Hintergrund die Zukunft von UWSF zielgruppengerecht gestalten zu können, bitten wir Sie, uns mithilfe eines bequemen und zeitsparenden Webformulars (max. 5 min) einige Fragen zur Struktur und Themenauswahl der Zeitschrift zu beantworten.

Bitte gehen Sie zur Onlineversion dieses freigeschalteten Beitrages (DOI: http://dx.doi.org/10.1007/s12302-009-0089-6), öffnen Sie den Link und setzen im Folgenden einfach Häkchen. Sie haben auch die Möglichkeit, freie Kommentare bei den ersten sechs Fragen zu formulieren:

http://www.surveymonkey.com/s.aspx?sm=ubGZq0ImD7d jxkTLMbZXYA_3d_3d

A. B. Heinrich $(\bowtie)$

Managing-Editor, Scientific Journals on behalf of Springer-Verlag,

Kirschgartenstr. 91, 69126 Heidelberg, Deutschland

E-Mail: ABH.scientificjournals@googlemail.com

H. Hollert

Editor-in-Chief UWSF, Institut für Umweltforschung -

Biologie V: Lehr- und Forschungsgebiet Ökosystemanalyse,

Worringerweg 1, 52074 Aachen, Deutschland

E-Mail: henner.hollert@bio5.rwth-aachen.de
Wir empfehlen Ihnen, das Internetformular zu benutzen, möchten Sie jedoch auch hier mit den Fragen vertraut machen.

1. Möchten Sie die jetzige Struktur der UWSF beibehalten, d.h. das Nebeneinander von Originalarbeiten, Diskussionsbeiträgen, Kommentaren, Übersichtsbeiträgen, Beitragsserien und aktuell informierenden Beiträgen wie die Corners der drei assoziierten wissenschaftlichen Gesellschaften (GDCh-Fachgruppe Umweltchemie und Ökotoxikologie, SETAC-GLB, Verband der Geoökologen in Deutschland VGöD)?

Ja

Nein

Wenn Sie die Frage verneinen, bitten wir um Ihre Begründung.

2. Würden Sie in jeder Ausgabe eine Gliederung in Beiträge für Hochschule, Industrie und Behörde empfehlen?

Ja

Nein

Wenn Sie die Frage verneinen, bitten wir um Ihre Begründung.

3. Entspricht die Themenauswahl Ihren Erwartungen?

Ja

Nein

Wenn Sie die Frage verneinen, bitten wir um Ihre Begründung.

4. Sehen Sie weiteren Themenbedarf?

Ja

Nein

5. In welchen Bereichen sehen Sie Themenbedarf? 
6. Welche Themenfelder finden Sie minder interessant?

7. Würden Sie empfehlen, die Zielgruppe der Studenten stärker zu berücksichtigen?

$\mathrm{Ja}$

Nein

8. Würden Sie empfehlen, neben den deutschsprachigen auch englischsprachige Beiträge zu akzeptieren?

Ja

Nein

9. Würden Sie empfehlen, die Zeitschrift ganz auf die englische Sprache umzustellen?
$\mathrm{Ja}$

Nein

10. Bitte formulieren Sie Ihre Anliegen, die in den neun Fragen nicht berücksichtigt worden sind und schließen Sie mit Ihrem Namen und dem Ihrer Gesellschaft (SETAC-GLB oder GDCh-FGUÖ oder VGöD).

Wir werden Ihre Antworten und Kommentare sorgfältig auswerten, danken für Ihre Unterstützung und grüßen Sie herzlich.

Prof. Dr. Henner Hollert, Herausgeber UWSF Almut B. Heinrich, Chefredaktion UWSF 